Europhysics Letters

PREPRINT

\title{
The Amplitude of Non-Equilibrium Quantum Interference in Metallic Mesoscopic Systems
}

\author{
C. Terrier, D. Babić(*), C. Strunk(**), T. Nussbaumer and C. Schönenberger \\ Institute of Physics, University of Basel, Klingelbergstrasse 82, CH-4056 Basel, Switzer- \\ land
}

PACS. 73.23.-b - Electronic transport in mesoscopic systems.

PACS. 72.10.-d - Theory of electronic transport; scattering mechanisms.

\begin{abstract}
. -
We study the influence of a DC bias voltage $V$ on quantum interference corrections to the measured differential conductance in metallic mesoscopic wires and rings. The amplitude of both universal conductance fluctuations (UCF) and Aharonov-Bohm effect (ABE) is enhanced several times for voltages larger than the Thouless energy. The enhancement persists even in the presence of inelastic electron-electron scattering up to $V \sim 1 \mathrm{mV}$. For larger voltages electronphonon collisions lead to the amplitude decaying as a power law for the UCF and exponentially for the ABE. We obtain good agreement of the experimental data with a model which takes into account the decrease of the electron phase-coherence length due to electron-electron and electron-phonon scattering.
\end{abstract}

If the size of a conductor is of order of the electron phase-coherence length $L_{\varphi}(T)$ the wave character of electrons leads to experimentally observable quantum interference contributions to the conductance $G$. These are the aperiodic and periodic fluctuations $\delta G$ of the conductance around its average value. In the former case, called universal conductance fluctuations, the interference pattern is formed as a superposition of contributions from a continuous range of possible interference paths. Averaged over either impurity configuration, magnetic field or energy, the root-mean-square (rms) conductance fluctuation $\delta G_{r m s}$ is of the order $\sim e^{2} / h$ [1]. The periodic fluctuations, known as the Aharonov-Bohm effect [2], are observed if the interference is imposed by geometry of a sample, most commonly in the form of a loop. If the loop is threaded by a magnetic flux $\phi, \delta G(\phi)$ exhibits periodic oscillations with a period $h / e$, and $\delta G_{r m s}$ is again $\sim e^{2} / h$. Both the UCF [3] and the ABE [4] are suppressed by ensemble averaging if independent phase-coherent units are connected in series. The above behaviour is characteristic of the linear-response regime, i.e. of $e V \ll k_{B} T$ or $e V \ll E_{c}$, where $E_{c}$ is the coherence energy (Thouless energy) determined by the size of the conductor. In non-equilibrium $\left(e V \gg E_{c}, k_{B} T\right)$ the fluctuations are expected to be remarkably different, as predicted theoretically by Larkin and Khmel'nitskiu (LK) [5]. If inelastic processes can be

$\left({ }^{*}\right)$ Present address: Department of Physics, Faculty of Science, University of Zagreb, Croatia.

$\left(^{* *}\right)$ Present address: Institute for Experimental and Applied Physics, University of Regensburg, Germany.

(C) EDP Sciences 
neglected the rms fluctuation $\delta g_{r m s}$ of the differential conductance $g$ increases with $V$ according to $\delta g_{r m s} \sim\left(e^{2} / h\right) \sqrt{V / V_{c}}$, where $V_{c}=E_{c} / e$. This, at first sight surprising result, can be understood as follows. At $V \gg V_{c}$ the relevant energy range for the transport subdivides into $N=V / V_{c}$ uncorrelated energy intervals, each contributing to the fluctuations of the current by an amount $\sim\left(e^{2} / h\right) V_{c}$. Incoherent superposition of these contributions leads to $\delta g_{r m s}$ being $N^{1 / 2}$ times larger than $e^{2} / h$. Inelastic scattering at large voltages destroys quantum interference. The enhancement of $\delta g_{r m s}(V)$ is thereby suppressed and $\delta g_{r m s}$ eventually decreases with increasing voltage [5]. Experimental studies of both the UCF [6, 0, 8, 9] and ABE [6, 10, 11] under non-equilibrium conditions have been done, but did not result in a satisfactory understanding of $\delta g(V)$. Moreover, [8] reported on a voltage-independent $\delta g_{r m s}$, [6] on a decrease of $\delta g_{r m s}(V)$ for $V>V_{c}$, whereas in [9, 10, 11] it was found that $\delta g_{r m s}(V)$ shows a non-monotonic behaviour. We also note that explanations [10, 11] of the non-monotonic behaviour of $\delta g(V)$, observed in the present work as well, were at a qualitative level.

In this paper we report on the non-equilibrium $\mathrm{UCF}$ and $\mathrm{ABE}$ in diffusive gold samples measured over a wide voltage range of $V \gg k_{B} T / e, V_{c}$ (strongly $V$-dominated energy averaging) that covers both the low-voltage $\left(V / V_{c}\right)^{1 / 2}$ enhancement of $\delta g_{r m s}$ and its suppression at large voltages. Emphasis is put on the decay of $\delta g_{r m s}^{U C F}(V)$ and $\delta g_{r m s}^{A B E}(V)$. It is shown that $\delta g_{r m s}(V)$ decays as a power law for the UCF and exponentially for the ABE. A quantitative comparison between simple models and the experiments allows to extract the voltagedependent phase-coherence length $L_{\varphi}(V)$ and to discuss the nature of inelastic scattering processes out of equilibrium.

The samples were produced by electron-beam lithography and evaporation of $99.99 \%$ pure gold. The substrate was silicon covered with $400 \mathrm{~nm}$ of $\mathrm{SiO}_{2}$. Three $20 \mathrm{~nm}$ thick samples of different planar geometries were prepared 12: (1) for the ABE measurements a ring of average diameter $1 \mu \mathrm{m}$ and line width $0.09 \mu \mathrm{m}$ (sample $\mathrm{S}_{A B E}$ ), with a resistance of $25.8 \Omega$ at $0.3 \mathrm{~K}$; (2) for the UCF measurements a $L_{w}=1.5 \mu \mathrm{m}$ long and $0.13 \mu \mathrm{m}$ wide wire (sample $\mathrm{S}_{U C F}$ ), with a resistance of $18.4 \Omega$ at $0.3 \mathrm{~K}$; (3) for the weak-localisation (WL) measurements a 98 $\mu \mathrm{m}$ long and $0.17 \mu \mathrm{m}$ wide wire (sample $\mathrm{S}_{W L}$ ). Sample $\mathrm{S}_{W L}$ was made relatively long in order to suppress the UCF. All samples were produced under identical conditions (the same source of gold and the same evaporation parameters). The diffusion constants $D$ are consequently very similar: $116 \mathrm{~cm}^{2} / \mathrm{s}$ for samples $\mathrm{S}_{U C F}$ and $\mathrm{S}_{W L}$, and $110 \mathrm{~cm}^{2} / \mathrm{s}$ for sample $\mathrm{S}_{A B E}$. The measurements were carried out in a ${ }^{3} \mathrm{He}$ cryostat with cryogenic rf-filtering, using a lowfrequency $(37 \mathrm{~Hz})$ lock-in technique to measure the differential conductance. Typical voltage resolution was $\sim 0.3 \mathrm{nV}$. Non-equilibrium $\mathrm{UCF}$ and ABE were measured at $T=300 \mathrm{mK}$ by superimposing a comparatively large DC bias voltage $V_{D C}$ to the small excitation voltage $V_{A C}$. The following hierarchy of energies was always maintained: $e V_{D C} \gg k_{B} T \geq e V_{A C}>E_{c}$. Ensemble averaging was achieved by measuring the UCF and ABE over a magnetic-field range of $\sim 2 \mathrm{~T}$, largely exceeding the correlation field. Typical sweep rates were $0.1 \mathrm{mT} / \mathrm{s}$. Fig.1a displays raw data of a differential-conductance measurement on sample $\mathrm{S}_{A B E}$, taken at $V_{D C}=0.5 \mathrm{mV}$. Both types of the fluctuations are present. In Fig.1b we show the Fourier transform of the same data, exhibiting a well-defined peak at the position corresponding to $\phi=h / e$. From the magnetoconductance traces we have extracted $\delta g_{r m s}^{A B E}$ and $\delta g_{r m s}^{U C F}$ for sample $\mathrm{S}_{A B E}$, as shown in Fig.2 (discussed in more detail later), and $\delta g_{r m s}^{U C F}$ for sample $\mathrm{S}_{U C F}$. The values of $\delta g_{r m s}^{U C F}$ have been determined as standard deviations of the whole differentialconductance traces. The amplitudes $\delta g_{r m s}^{A B E}$ have been calculated by averaging the periodic part of the fluctuations over $\sim 500$ periods, after the coarse background has been removed numerically.

The WL was measured on sample $\mathrm{S}_{W L}$ in the linear-response regime $\left(V_{D C}=0\right)$ and in the temperature range $0.3-10 \mathrm{~K}$. By fitting a one-dimensional WL expression [13] to the low-field 
magnetoconductance data we obtained the linear-response $L_{\varphi}$ as a function of temperature, as shown in the inset to Fig.3. Similarly to published work 14, 15, $L_{\varphi}(T)$ saturates at low temperatures (below $1 \mathrm{~K}$ ), which has been attributed to impurity mediated inelastic electronelectron scattering [16]. At high temperatures $L_{\varphi}$ follows a $L_{\varphi} \propto T^{-q}$ dependence with $q \approx 1.2$ that suggests dephasing by electron-phonon interaction. At $T=300 \mathrm{mK}, L_{\varphi}=3 \mu \mathrm{m}$ and $\tau_{\varphi}=\sqrt{L_{\varphi}^{2} / D}=0.77 \mathrm{~ns}$, implying that in the linear-response regime the diffusion of electrons is coherent throughout the samples $S_{U C F}$ and $S_{A B E} . L_{\varphi}$ has to be compared further with the effective length of the sample $L_{c 0}$ which depends on the particular geometry and the coherence phenomenon investigated. For the UCF in sample $\mathrm{S}_{U C F}, L_{c 0}=L_{w}=1.5 \mu \mathrm{m}$. In the case of ABE in sample $\mathrm{S}_{A B E}, L_{c 0}=C_{r} \approx 3.14 \mu \mathrm{m}$, where $C_{r}$ is the circumference of the ring. Finally, for the UCF in sample $\mathrm{S}_{A B E}, L_{c 0}$ is calculated as the distances ring - voltage contacts plus $C_{r} / 2$, which gives $\approx 2.2 \mu \mathrm{m}$. Thus, $L_{\varphi}(300 \mathrm{mK})$ is always bigger or very similar to $L_{c 0}$, so that the linear-response coherence energy is set by the time of electron diffusion through the sample according to $E_{c 0}=e V_{c 0}=\hbar D / L_{c 0}^{2}$.

With increasing temperature or applied voltage the coherence length decreases, and once it becomes shorter than $L_{c 0}$ the interference contributions are suppressed. The effect of temperature was demonstrated by Milliken et al. [17]. Their method was to determine $L_{\varphi}(T)$ from the WL and to use this to describe the decay of equilibrium $\delta G$, which turned out to be a power law for the UCF and exponential for the ABE. Our approach is complementary: we keep the bath temperature constant and investigate the voltage dependence of the nonequilibrium $\delta g$. While Milliken et al. observed a monotonically-decreasing $\delta G(T)$, in our case $\delta g(V)$ is a non-monotonic function, as shown in Fig.2. For small $V_{D C}$ (but still much larger than $\left.V_{c 0}\right), \delta g_{r m s}$ is enhanced by $V_{D C}$. At higher voltages $\delta g_{r m s}$ decreases with $V_{D C}$, faster for the $\mathrm{ABE}$ than for the $\mathrm{UCF}$, which is in qualitative agreement with the results of Milliken et al.

Let us first turn to the details of non-equilibrium UCF. For convenience we denote the phase-coherence length at a finite bias voltage by $L_{\varphi}(V)$, while $L_{\varphi}(T)$ referes to the linearresponse value. If $V_{D C} \gg V_{c 0}, k_{B} T / e$ and $L_{\varphi}\left(V_{D C}\right)>L_{c 0}$, the LK enhancement of $\delta g_{r m s}$ holds and one expects that $\delta g_{r m s} \propto \sqrt{V_{D C} / V_{c 0}}$. Once $L_{\varphi}\left(V_{D C}\right)$ becomes substantially smaller than $L_{c 0}$ inelastic processes start to be important for most of the electrons, and the sample effectively subdivides into $L_{c 0} / L_{\varphi}$ uncorrelated phase-coherent sections in series. The LK enhancement in a single section is still valid if the voltage drop $V_{D C}^{s e c}=V_{D C}\left(L_{\varphi} / L_{c 0}\right)$ over a section exceeds the corresponding coherence voltage $V_{c}^{s e c}=V_{c 0}\left(L_{c 0} / L_{\varphi}\right)^{2}$. Following the LK arguments, $\delta g_{r m s}$ corresponding to a single section is proportional to $\left(V_{D C}^{s e c} / V_{c}^{s e c}\right)^{1 / 2}=$ $\left[\left(V_{D C} / V_{c 0}\right)\left(L_{\varphi} / L_{c 0}\right)^{3}\right]^{1 / 2}$. Incoherent addition of these contributions of sections in series leads to the differential conductance being suppressed by a factor $\left(L_{\varphi} / L_{c 0}\right)^{1 / 2}$, and hence

$$
\delta g_{r m s}^{U C F}=A_{U C F} \frac{e^{2}}{h} \sqrt{\frac{V_{D C}}{V_{c 0}}}\left(\frac{L_{\varphi}\left(V_{D C}\right)}{L_{c 0}}\right)^{2},
$$

where $A_{U C F}$ is a constant prefactor. This expression is different from that resulting from the linear-response ensemble averaging, and valid if $V_{D C}>V_{c 0}$ and $L_{\varphi}<L_{c 0}$ [18]. It enables an unambiguous determination of $L_{\varphi}\left(V_{D C}\right)$. Since the form of $L_{\varphi}\left(V_{D C}\right)$ is not known a priori, we cannot determine $A_{U C F}$ and $L_{\varphi}\left(V_{D C}\right)$ simultaneously from a direct fit to the data. To avoid this problem we fix $A_{U C F}$ by setting $L_{\varphi}=L_{c 0}$ for the first measured point which satisfies $V_{D C} \gg V_{c 0}$, and then simply extract $L_{\varphi}\left(V_{D C}\right)$ for all the other points from the measured data. For the two sets of UCF data, i.e. for samples $\mathrm{S}_{U C F}$ and $\mathrm{S}_{A B E}$, we have obtained excellent agreement in $L_{\varphi}\left(V_{D C}\right)$, as we show in Fig.3 by open $\left(\mathrm{S}_{A B E}\right)$ and full $\left(\mathrm{S}_{U C F}\right)$ squares. Normalised to the corresponding values of $L_{c 0}$ both results collapse onto a single 
curve. The phase-coherence length deduced from our non-equilibrium experiment $L_{\varphi}(V)$ has a qualitatively similar functional form as $L_{\varphi}(T)$ obtained from the WL measurements (apart from the saturation of $L_{\varphi}(T)$, since saturation of $L_{\varphi}(V)$ is not expected for $e V \gg k_{B} T$ [16]). Two regimes with different power-law dependences are discernible. In the region of increasing $\delta g_{r m s}\left(V_{D C}\right)$ there is a power law $L_{\varphi}\left(V_{D C}\right) \propto V_{D C}^{-s}$, where $s=0.19 \pm 0.02$. For decreasing $\delta g_{r m s}\left(V_{D C}\right)$, i.e. where $L_{\varphi}\left(V_{D C}\right)$ is considerably smaller than $L_{c 0}$, the dependence changes to $L_{\varphi} \propto V_{D C}^{-p}$ with $p=0.57 \pm 0.03$. Inserting thus found $L_{\varphi}$ back into Eq.1 results in the dotted line in Fig.2.

We now discuss the non-equilibrium ABE. Here the LK theory cannot be directly applied to extract $\delta g_{r m s}$. Subdivision of a ring into phase-coherent sections makes no sense in this case, as only those electrons which stay coherent over the whole length $C_{r}$ contribute to the interference. However, since the same physics governs the UCF and ABE, $\delta g_{r m s}^{A B E}$ is expected to be $\propto \sqrt{V_{D C} / V_{c 0}}$ in the regime where inelastic scattering is absent. This is indeed the case, as shown in Fig.2. The form of the suppression of $\delta g_{r m s}^{A B E}$ can be inferred from a theoretical analysis of DiVincenzo and Kane [19]. They have shown that inelastic processes influence the $\mathrm{ABE}$ in two ways. First, the fluctuations are suppressed as $\exp \left(-\beta C_{r} / L_{\varphi}\right)$, where $\beta$ is of order unity in the range $1<C_{r} / L_{\varphi}<3$ and smaller in the case of a true exponential decay occurring in the asymptotic limit $C_{r} / L_{\varphi} \rightarrow \infty$. Second, the proper form of $E_{c}$ in the presence of inelastic processes is given by $E_{c}^{i n}=\hbar D / C_{r}^{2-\alpha} L_{\varphi}^{\alpha}=E_{c 0}\left(C_{r} / L_{\varphi}\right)^{\alpha}$ with $\alpha \approx 1.3$. This renormalisation of the coherence energy is a consequence of the statistics for those electrons that diffuse around a ring without being scattered inelastically [19]. Roughly, the probability distribution for an electron to contribute to the $\mathrm{ABE}$ has a maximum at a value of the electron traversal time $\sim D / C_{r} L_{\varphi}[19]$. Combining the above arguments we can write for $L_{\varphi}<C_{r}$ :

$$
\delta g_{r m s}^{A B E}=A_{A B E} \frac{e^{2}}{h} \sqrt{\frac{V_{D C}}{V_{c 0}}}\left(\frac{L_{\varphi}\left(V_{D C}\right)}{C_{r}}\right)^{0.65} e^{-\beta C_{r} / L_{\varphi}\left(V_{D C}\right)} .
$$

Good agreement of the ABE data with both Eq.2 and the UCF data is obtained for $\beta \approx 1.1$, as we show in Fig. 2 by the solid curve and in Fig. 3 by crosses.

Below we discuss how the observed power law dependences in $L_{\varphi}\left(V_{D C}\right)$ can be linked to microscopic scattering processes. From Figs.2,3 we can distinguish two regimes (denoted by I and II in Fig.3): (I) $\delta g_{r m s}\left(V_{D C}\right)$ increases with $V_{D C}$ and $L_{\varphi}\left(V_{D C}\right) \propto V_{D C}^{-0.19}$, and (II), $\delta g_{r m s}\left(V_{D C}\right)$ decreases with $V_{D C}$ while $L_{\varphi}\left(V_{D C}\right) \propto V_{D C}^{-0.57}$.

The phase-coherence length is determined by the dephasing rate $\tau_{\varphi}^{-1}$ via $L_{\varphi}=\sqrt{D \tau_{\varphi}}$. $\tau_{\varphi}^{-1}$ can be expressed as an average of the inelastic scattering rate $\tau_{i n}^{-1}(\epsilon),(\epsilon$ is the energy exchanged in the interaction) of electrons over the accessible energy range given by the width of the electron distribution function $f$ [15]. If the electron-electron scattering is dominant, the scattering rate is energy dependent as described by the kernel function $K(\epsilon)=\kappa_{\eta} \epsilon^{-\eta}$. The energy interval $E$ accessible for the scattering is set by $k_{B} T$ in equilibrium and $e V_{D C}$ in nonequilibrium. At high energies, where single scattering events determine $\tau_{\varphi}$, a simple argument [15] leads to $\tau_{\varphi, e e}^{-1}(E) \propto E^{1 / \eta}$ [20]. Two choices for $\eta$ are currently under debate: $\eta=3 / 2$ for the disorder enhanced Coulomb interaction [15] and $\eta=2$ for magnetic impurity mediated interaction 16, 21]. This results in $L_{\varphi, e e}^{-1}\left(V_{D C}\right) \propto V_{D C}^{1 / 3}$ for $\eta=3 / 2$, and $L_{\varphi, e e}^{-1}\left(V_{D C}\right) \propto V_{D C}^{1 / 4}$ for $\eta=2$. Hence the kernel exponent $\eta=2$ appears more consistent with our experimental observation of $L_{\varphi}^{-1} \propto V_{D C}^{0.19}$. This is supported by the observed saturation of the equilibrium $\tau_{\varphi}$ for temperatures below $1 \mathrm{~K}$.

We emphasise that - independently of the precise interaction mechanism - the rather weak decrease of $L_{\varphi, e e}$ with increasing $V$ is insufficient to suppress the LK enhancement of $\delta g_{r m s}$. This is seen as follows. We recall that the condition for the appearance of the LK 
enhancement in a phase-coherent section of the wire is $V_{D C}^{s e c}>V_{c}^{s e c}$, which can be written as $V_{D C} / V_{c 0}>\left(L_{c 0} / L_{\varphi}\right)^{3}$. Since $L_{\varphi, e e} \propto V_{D C}^{-1 / 4}$, the right-hand side of the above inequality increases more slowly with $V_{D C}$ than the left-hand side, and the condition for the enhancement is maintained over the whole voltage range of increasing $\delta g_{r m s}\left(V_{D C}\right)$.

While the regime $I$ is determined by electron-electron scattering and a non-equilibrium electron distribution function $f$, the stronger decay of $L_{\varphi}(V)$ in the regime II is caused by electron-phonon scattering. It can be very well described by equilibrium properties if we assume local thermal equilibrium with an elevated electron temperature $T_{e l}\left(V_{D C}\right)$. Electronphonon scattering leads to $\tau_{\varphi, e p} \propto T_{e}^{-m}$, where $m$ equals 3 for very clean samples, 4 for strongly disordered samples, and $2-3$ for samples of intermediate degree of disorder [22]. Since $T_{e}$ is related to the applied voltage by $T_{e} \propto V^{2 /(2+m)}$ [23], one obtains $\tau_{\varphi, e p} \propto V^{-2 m /(2+m)}$ and $L_{\varphi, e p} \propto V^{-m /(2+m)}$. Our result $L_{\varphi}\left(V_{D C}\right) \propto V_{D C}^{-0.57 \pm 0.03}$ gives $m=2.6 \pm 0.3$, which agrees well with the value 2.5 obtained in a noise measurement on similar samples [24]. Moreover, our WL result $\left(L_{\varphi, e p}(T) \propto T^{-m / 2}\right)$ is $m=2.4$, in excellent agreement with the above values. We also note that even though $T_{e}$ rises up to $\sim 10-15 \mathrm{~K}$ at high $V_{D C}$ the energy averaging given by the LK approach remains essentially unaffected because $T_{e}$ increases more slowly than the voltage itself. Although our phenomenological analysis leads to a consistent interpretation of our data, further theoretical work is needed to fully understand the dephasing in the case of strong non-equilibrium.

In conclusion, we have measured the universal conductance fluctuations and AharonovBohm effect in mesoscopic gold samples under highly non-equilibrium conditions of large applied bias voltages. The rms fluctuation $\delta g_{r m s}$ of the differential conductance initially increases with voltage $\propto \sqrt{V / V_{c}}$, which demonstrates the validity of the theoretical prediction by Larkin and Khmel'nitskiu. This increase is followed by a decay of $\delta g_{r m s}$ at higher voltages, where inelastic scattering becomes substantial. The amplitude decays as a power law for the universal conductance fluctuations and exponentially in the case of the Aharonov-Bohm effect. The decrease of the phase-coherence length with increasing voltage is in good agreement with the inferred $L_{\varphi}(V)$ dependences for electron-electron and electron-phonon scattering. In particular, the electron-electron collisions are not sufficient to suppress the enhancement mechanism. This work was supported by the Swiss National Science Foundation.

\section{REFERENCES}

[1] Lee P. A. and Stone D., Phys. Rev. Lett, 55 (1985) 1662.

[2] Aharonov Y. and Bohm D., Phys. Rev., 115 (1959) 485.

[3] Skocpol W. J., Mankiewich P. M., Howard R. E., Jackel L. D., Tennant D. M. and Stone A. D., Phys. Rev. Lett., 56 (1986) 2865.

[4] Umbach C. P., Van Haesendonck C., Laibowitz R. B., Washburn S., and Webb R. A., Phys. Rev. Lett., 56 (1986) 386.

[5] Larkin A. I. and Khmel'nitskĭ D. E., Pis'ma Zh. Eksp. Teor. Fiz., 91 (1986) 1815 [Sov. Phys. JETP, 64 (1986) 1075]; Phys. Scr., T14 (1986) 4.

[6] Webb R. A. ,Washburn S. and Umbach C. P., Phys. Rev. B, 37 (1988) 8455.

[7] Kaplan S. B., Phys. Rev. B, 38 (1988) 7558.

[8] Ralph D. C., Ralls K. S. and Buhrman R. A., Phys. Rev. Lett., 70 (1993) 986.

[9] Schäfer R., Hecker K., Hegger H. and Langheinreich W., Phys. Rev. B, 53 (1996) 15964.

[10] Terrier C., Strunk C., Nussbaumer T., Babić D. and Schönenberger C., Fizika A (Zagreb), 8 (1999) 157.

[11] Häussler R., Weber H. B. and von Löhneysen H., J. Low Temp. Phys, 118 (2000) 467.

[12] The results for these samples are representative of the data obtained by measurements carried 

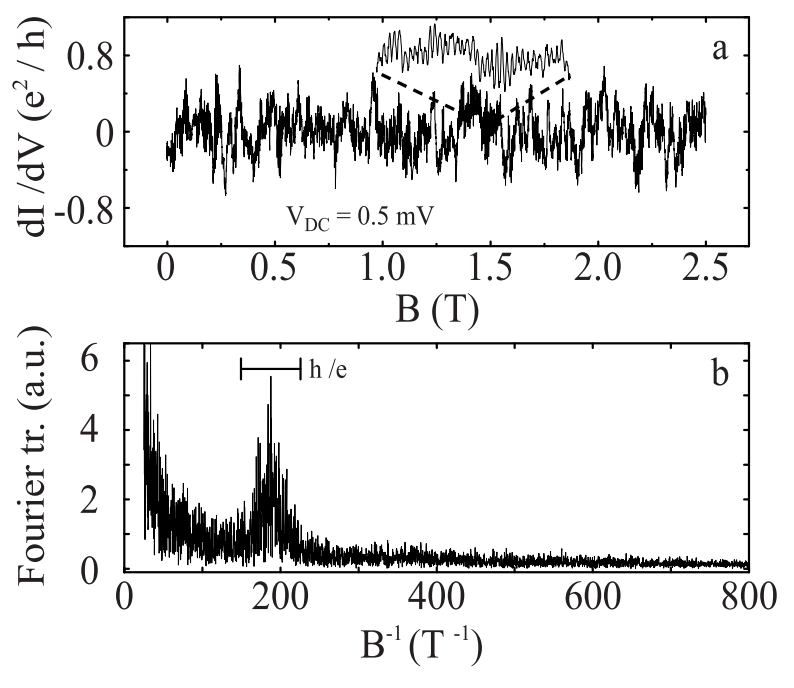

Fig. 1 - (a) A differential-magnetoconductance trace of sample $\mathrm{S}_{A B E}$, taken at $V_{D C}=0.5 \mathrm{mV}$. Both the periodic (expanded view) and aperiodic fluctuations are present. (b) Fourier transform of (a), showing a well-defined peak at the position corresponding to $\phi=h / e$.

out on altogether eleven samples. We did not find any significant sample dependence of the results.

[13] Al'tshuler B. L. and Aronov A. G., Pis'ma Zh. Teor. Eksp. Teor. Fiz., 33 (1981) 515 [JETP Lett, 33 (1981) 499].

[14] Mohanty P., Physica B, 280 (2000) 446.

[15] Goughm A. B.,Pierre F., Pothier H., Esteve D. and Birge N. O., J. Low Temp. Phys.,

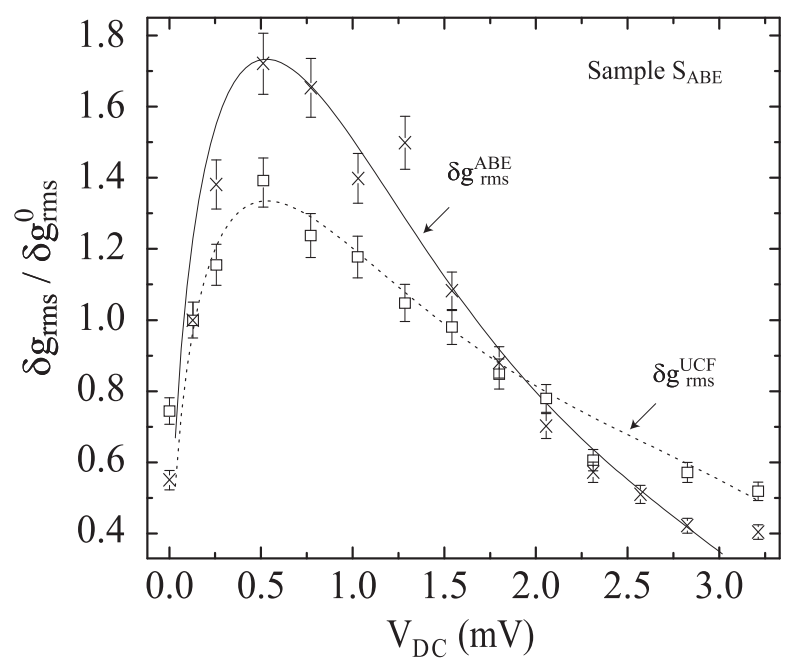

Fig. 2 - Plot of $\delta g_{r m s}^{A B E}$ (crosses) and $\delta g_{r m s}^{U C F}$ (open squares) vs. $V_{D C}$ for sample $\mathrm{S}_{A B E}$, with $\delta g_{r m s}$ normalised to the values $\delta g_{r m s}^{0}$ at the first $V_{D C} \neq 0$ points (which also satisfy the criterion $V_{D C} \gg$ $V_{c 0}$ ). The lines are plots of Eq.1 (dashed line) and Eq.2 (solid line) discussed in the text. 
C. Terrier, D. Babić, C. Strunk, T. Nussbaumer and C. Schönenberger: The Amplitude Of Non-Equilibrium Quantum I

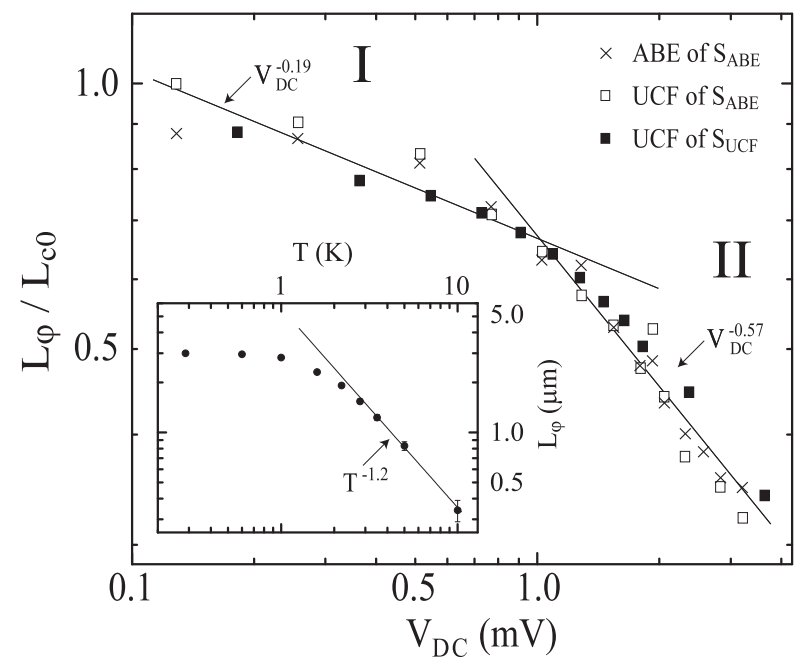

Fig. 3 - Phase-coherence length $L_{\varphi}\left(V_{D C}\right)$, normalised to the characteristic lengths $L_{c 0}$ defined in the text, deduced from the non-equilibrium $\mathrm{UCF}$ and $\mathrm{ABE}$ measurements. Crosses: ABE in sample $\mathrm{S}_{A B E}$. Open squares: $\mathrm{UCF}$ in sample $\mathrm{S}_{A B E}$. Full squares: UCF in sample $\mathrm{S}_{A B E}$. Below $V_{D C} \sim 1$ $\mathrm{mV}, L_{\varphi} \propto V_{D C}^{-0.19 \pm 0.02}$, and above $L_{\varphi} \propto V_{D C}^{-0.57 \pm 0.03}$. Inset: Linear-response coherence length $L_{\varphi}(T)$ found from the WL measurements on sample $\mathrm{S}_{W L}$.

118 (2000) 447.

[16] Kaminski A. and Glazman L. I., Phys. Rev. Lett., 86 (2001) 2400.

[17] Milliken F. P., Washburn S., Umbach C. P., Laibowitz R. B. and Webb R. A., Phys. Rev. B, 36 (1987) 4465.

[18] Strictly speaking, the conditions are $V_{D C} \gg V_{c 0}$ and $L_{\varphi} \ll L_{c 0}$.

[19] DiVincenzo D. P. and Kane C. L., Phys. Rev. B, 38 (1988) 3006.

[20] The saturation of $\tau_{\varphi}(T)$ at very low energies is not covered by the simple argument of Ref. 15.

[21] Pierre F., Pothier H., Esteve D., Devoret M. H., Gougam A. B. and Birge N. O., in Proceedings of the NATO Advanced Research Workshop on Size-Dependent Magnetic Scattering, Pecs, Hungary (Kluwer, Dodrecht, The Netherlands) 2001

[22] Schmid A., in Localization, Interaction and Transport Phenomena, edited by Kramer B., Bergmann G. and Bruynsereade Y. (Springer, Berlin) 1985

[23] Anderson P. W., Abrahams E. and Ramakrishnan T. V., Phys. Rev. Lett., 43 (1979) 718.

[24] Henny M., PhD Thesis, University of Basel, 1998. 